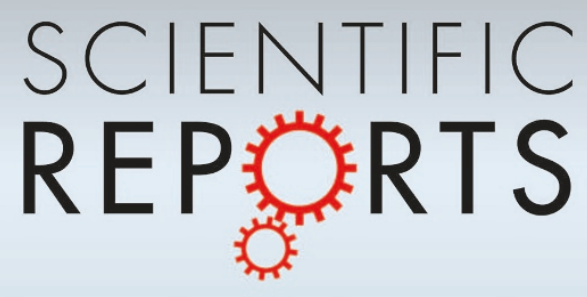

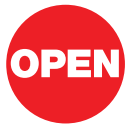

SUBJECT AREAS:

TRANSLATION

GENE REGULATION

TRANSCRIPTION

CELLULAR MICROBIOLOGY

Received

26 July 2012

Accepted

15 October 2012

Published

14 November 2012

Correspondence and requests for materials should be addressed to

R.Z. (rzhang@med. wayne.edu)

\section{Hkat, a novel nutritionally regulated transmembrane protein in adipose}

\section{tissues}

\author{
Ren Zhang
}

Center for Molecular Medicine and Genetics, School of Medicine, Wayne State University, Detroit 48201, USA.

White adipose tissue is an active endocrine organ regulating many aspects of whole body physiology and pathology. Adipogenesis, a process in which premature cells differentiate into adipocytes, is a complex process that includes orchestrated changes in gene expression and cell morphology in response to various nutritional and hormonal stimuli. To profile transcriptome changes in response to nutritional stimulation, we performed RNA-seq on fat in mice treated with either a high-fat diet or fasting. We identified a novel nutritionally regulated gene, Gm12824, named Hkat (heart, kidney, adipose-enriched transmembrane protein). We show that both fasting and obesity dramatically reduce $H k$ at in white adipose tissue, and that fasting reduces while obesity increases its expression in brown fat. Hkat is localized to the plasma membrane and induced during adipogenesis. Therefore, Hkat is a novel nutritionally regulated gene that is potentially involved in metabolism.

W hite adipose tissue, the major energy reservoir in higher eukaryotes, plays a central role in maintaining energy homeostasis ${ }^{1-6}$. During periods of nutritional abundance, white adipose tissue increases uptake of glucose and fatty acids and stores them in the form of triglycerides, which are released in the form of free fatty acids and glycerol during nutritional deprivation.

Adipose tissue secretes numerous cytokines, called adipokines, which are involved in regulating insulin sensitivity, energy expenditure, food intake, blood pressure and inflammatory responses. Some examples of these adipokines include Leptin ${ }^{7}$, Adiponectin ${ }^{8-10}$, Resistin ${ }^{11}$ and $\mathrm{TNF} \alpha^{12}$. Expansion of white adipose tissue, a hallmark of obesity, is a major risk factor for various pathological conditions, such as type 2 diabetes, hypertension and cardiovascular diseases ${ }^{13-15}$. Conversely, absence of adipose tissue, lipodystrophy, also exhibits diabetes phenotypes ${ }^{16,17}$.Therefore, although traditionally adipose tissue was regarded as an inactive energy reservoir only; the past two decades have seen a dramatic increase in our knowledge about adipocyte biology, indicating that adipose tissue is an active endocrine organ regulating many aspects of whole body physiology and pathology ${ }^{18-22}$.

Adipogenesis, a process in which premature cells differentiate into adipocytes, is a complex process that includes orchestrated changes in gene expression and cell morphology in response to various nutritional and hormonal stimuli. Adipogenesis relies on the accurate regulation of a complicated cascade of transcription factors, such as peroxisome proliferator-activated receptor gamma (PPAR $\gamma$ ), members of the CCAAT/enhancer-binding proteins (C/EBPs), and sterol regulatory element-binding protein 1 (SREBP1) ${ }^{23-31}$. Identification of novel genes and pathways in influencing adipocyte differentiation is helpful to understand the pathology of metabolic syndromes, and this is highlighted by the current obesity epidemic ${ }^{32,33}$.

To profile transcriptome changes in response to nutritional stimulation, we performed RNA-seq experiments on fat in mice treated with either a high-fat diet or fasting. We identified a novel nutritionally regulated gene, Gm12824, named Hkat (heart, kidney, adipose-enriched transmembrane protein). We show that both fasting and obesity dramatically reduce Hkat in white adipose tissue. Fasting reduces and obesity increases its expression in brown fat. Hkat, a transmembrane protein, is induced during adipogenesis, and it is therefore a novel nutritionally regulated gene that is potentially involved in adipogenesis.

\section{Results}

Hkat is evolutionarily conserved. To comprehensively identify nutritionally regulated genes, we performed RNA-seq experiments on white adipose tissue, in mice treated with 3-month HFD or 24-hour fasting. Here, we focus on the analysis of the novel gene Hkat, which was one of the identified genes that were sensitive to nutritional stimulation. The symbol for mouse Hkat is Gm12824, and its human homolog is LOC388630 (Table 1). 


\begin{tabular}{|lll|}
\hline Table 1 | IDs of Hkat in databases & \multicolumn{1}{c|}{ Mouse } & \multicolumn{1}{c|}{ Human } \\
\cline { 2 - 3 } Name & \multicolumn{1}{c}{ Hkat } & \multicolumn{1}{c|}{ HKAT } \\
\hline Symbol & Gm12824 & LOC388630 \\
Chromosome & 4 & 1 \\
Location (bp) & $114079329-114287703$ & $48226200-48462562$ \\
RefSeq & NP_001079018 & NP_001181915 \\
Ensembl & ENSMUSG00000070867 & ENSG00000204018 \\
Entrez & 666048 & 388630 \\
Uniprot & B 1ATG9 & A6NFA1 \\
\hline
\end{tabular}

Hkat is evolutionarily conserved. A Blast search against the nonredundant protein database at National Center for Biotechnology showed that Hkat has homologues in multiple species, including D. rerio and C. elegans (Fig. 1A). Between protein sequences of human and mouse, $85 \%$ of residues were identical, whereas between those of human and chicken, $81 \%$ of residues were identical (Fig. 1B), indicating that Hkat is highly evolutionarily conserved.

Both the human and mouse Hkat have 517 amino acids in length. The program SignalP 4.0 predicted Hkat has a signal peptide from the residue 1-19. According to CDD, a conserved domain database, Hkat harbors a conserved domain of TraB from the residue 52-348 (Fig. 1C). TraB is a protein family that is involved in production of peptide pheromones by bacteria. It is unclear what the functions of TraB are in mammalian species. In addition, Hkat also harbors a transmembrane domain from the residue 495-515. Because of the presence of a signal peptide, it is likely that the N-terminal of Hkat is in extracellular space, and the C-terminal is in the cytoplasm, connected by the transmembrane domain (Fig. 1C).

Hkat is nutritionally regulated in fat. To examine whether Hkat is nutritionally regulated, we treated mice with fasting or HFD, and then examined Hkat expression by qPCR analysis. In white adipose
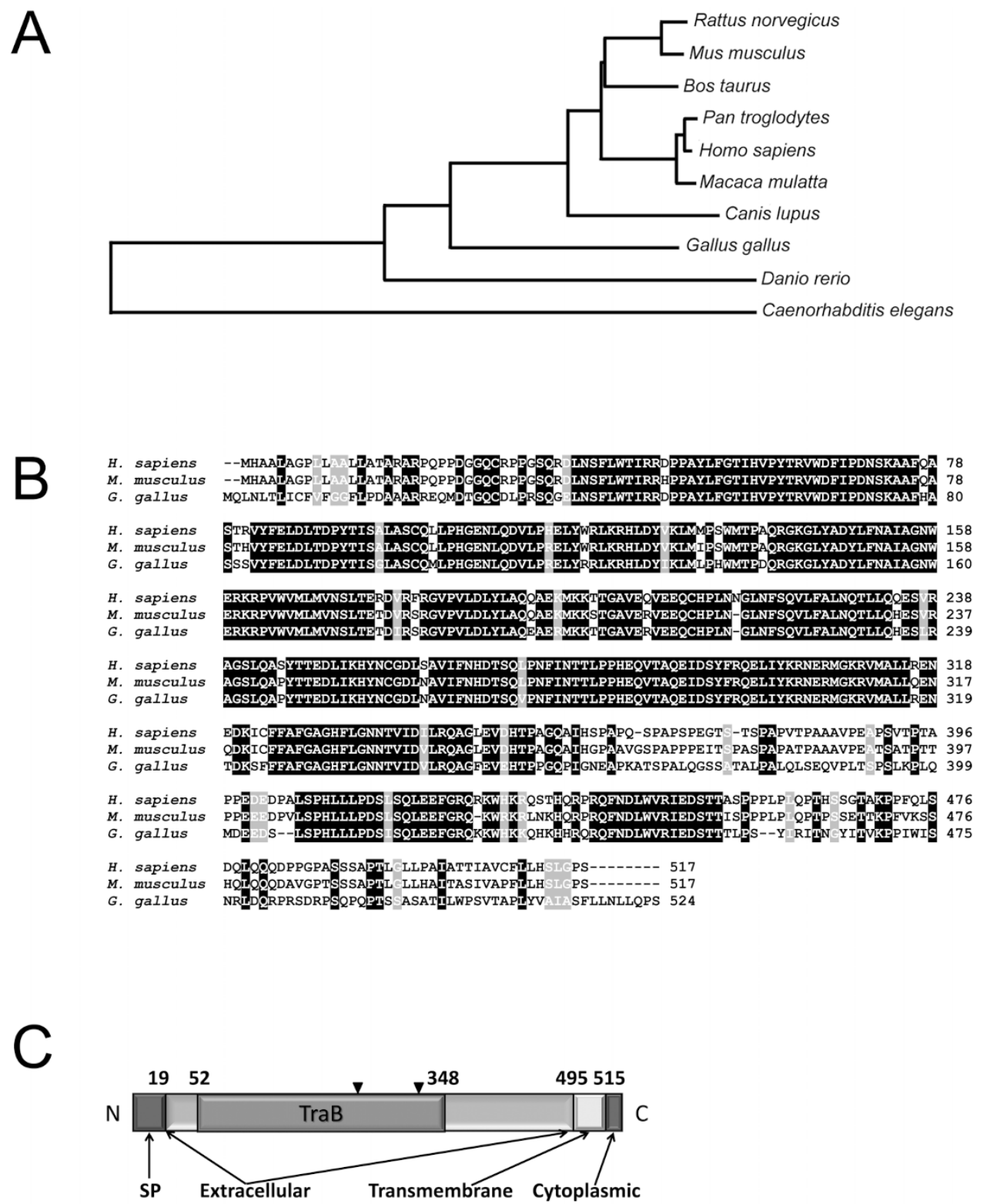

Figure 1 The Hkat protein is evolutionarily conserved. A) The evolutionary tree of Hkat orthologs. B) Alignment of Hkat protein sequences of human, mouse and chicken. Between human and mouse, $85 \%$ of residues are identical, whereas between human and chicken, $81 \%$ of residues are identical. C) Hkat has a signal peptide (SP), extracellular domain, transmembrane domain and cytoplasmic region, and it has a domain that belongs to TraB family. Triangle denotes predicted glycosylation. 
tissue (WAT), 24-hour fasting reduced Hkat expression for more than $80 \%(\mathrm{P}<0.01)$, and refeeding 4 hours following the fasting normalized its expression (Fig. 2A). In mice with 3-month of HFD treatment, Hkat expression in WAT was reduced for more than $70 \%$ $(\mathrm{P}<0.01)$ (Fig. 2B). In brown adipose tissue (BAT), fasting reduced Hkat expression for about $70 \%(\mathrm{P}<0.01)$, which was normalized by refeeding (Fig. 2C). Conversely, Hkat expression in BAT was increased for about twofold in mice with diet induced obesity $(\mathrm{P}<$ 0.01) (Fig. 2D). That is to say, fasting reduces Hkat expression in both WAT and BAT. HFD treatment suppressed its expression in WAT, while increased its expression in BAT. Therefore, Hkat expression is nutritionally regulated in fat.

Hkat is abundant in fat, but highest expressed in heart and kidney. Tissue expression pattern for a novel gene can be informative in revealing its functions. We therefore examined the expression pattern of Hkat in various mouse tissues. Three male C57B6 mice were used to dissect various tissues, including hypothalamus, cortex, stomach, small intestine, large intestine, colon, liver, heart, blood vessel, kidney, spleen, lung, muscle, urinary bladder, testis and fat. The fat tissues included epididymal fat, subcutaneous fat and brown fat. Hkat expression is highest in heart and kidney. In addition, Hkat is abundant in all fat depots, including perigonadal fat, subcutaneous fat and brown fat (Fig. 3A).

Hkat is localized to the plasma membrane. Proteins need to localize to specific subcellular compartments to perform functions, therefore the subcellular localization of a protein is critical to reveal its functions. The Universal Protein Resource annotates the protein as having a transmembrane domain, from amino acid 495 to 515 (Fig. 1C). To gain experimental evidence, we examined the subcellular localization by fluorescent protein imaging. A fusion protein with a GFP at the C-terminal of Hkat was made by cloning the Hkat open reading frame into a vector containing the GFP gene. The vector encoding the fusion protein Hkat-GFP was then cotransfected with a vector encoding red fluorescent protein fused with LCK (lymphocyte-specific protein tyrosine kinase), which is localized to the plasma membrane. Indeed, red signals, which indicate the location of LCK, localized to the plasma membrane (Fig. 3B). Green signals, which indicate the location of Hkat, also localized to the plasma membrane (Fig 3C). Note that because the vectors was transfected into the cells via transient transfection, some cells had Hkat-GFP only (green signals only), while some cells had LCK-RFP only (red signals only). Only cells having the two plasmids at the same time showed co-localization. Merging the two signals shows that in some cells Hkat and LCK co-localized (Fig. 3D), indicating that Hkat is localized to the plasma membrane in these cells.

Hkat is transiently induced during adipogenesis. Hkat is abundantly expressed in WAT, and its expression in WAT is sensitive to nutritional stimulation. This makes it possible that Hkat plays a functional role in WAT. We therefore examined whether Hkat is induced during adipogenesis. 3T3 L1 cells were differentiated into mature adipocytes. Two days after reaching confluence, cells were cultured in differentiation medium that contained insulin and
A

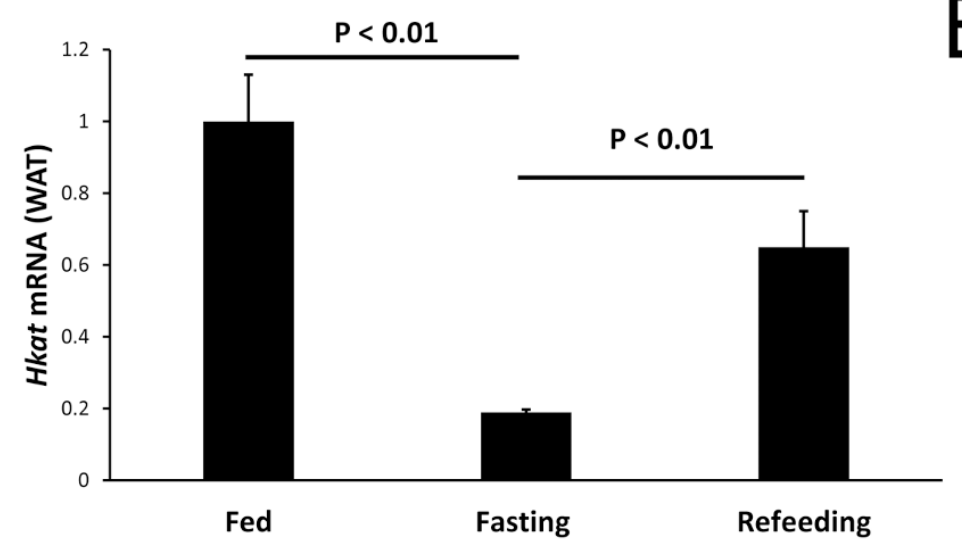

C

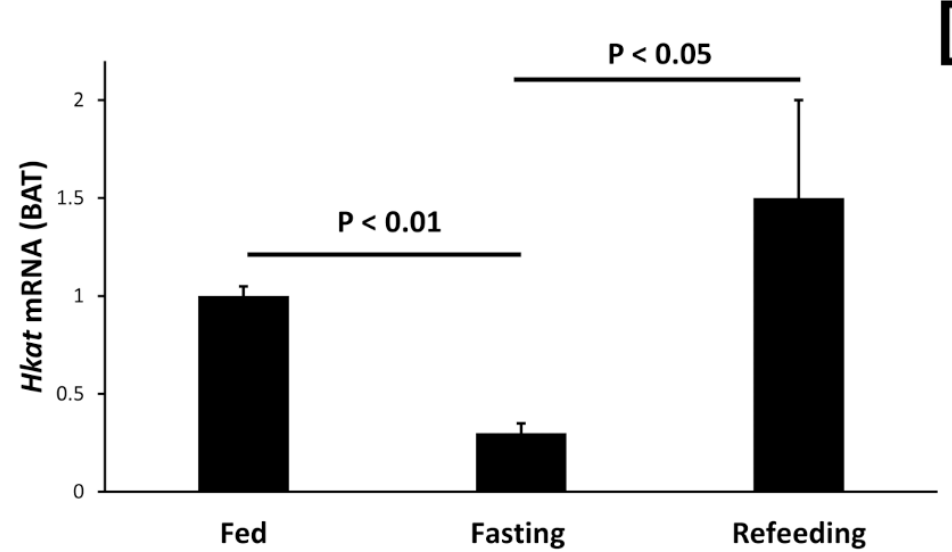

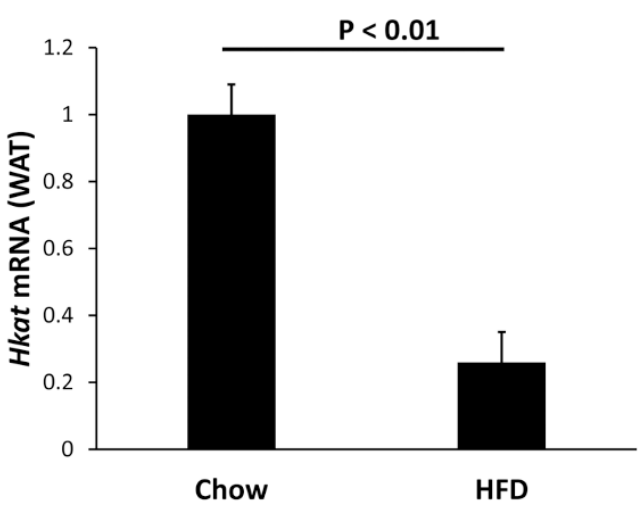

D

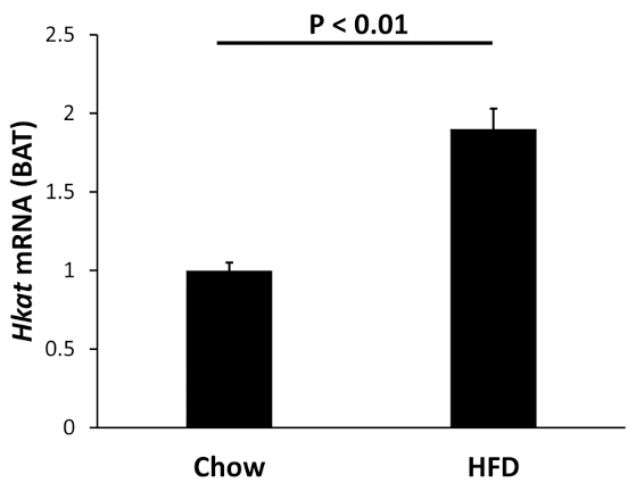

Figure $2 \mid$ Hkat in fat is nutritionally regulated. A) Fasting and B) HFD treatment reduced Hkat expression in fat. C) Fasting reduced Hkat expression while D) HFD treatment increased its expression in brown fat. Data are represented as mean \pm SEM. 

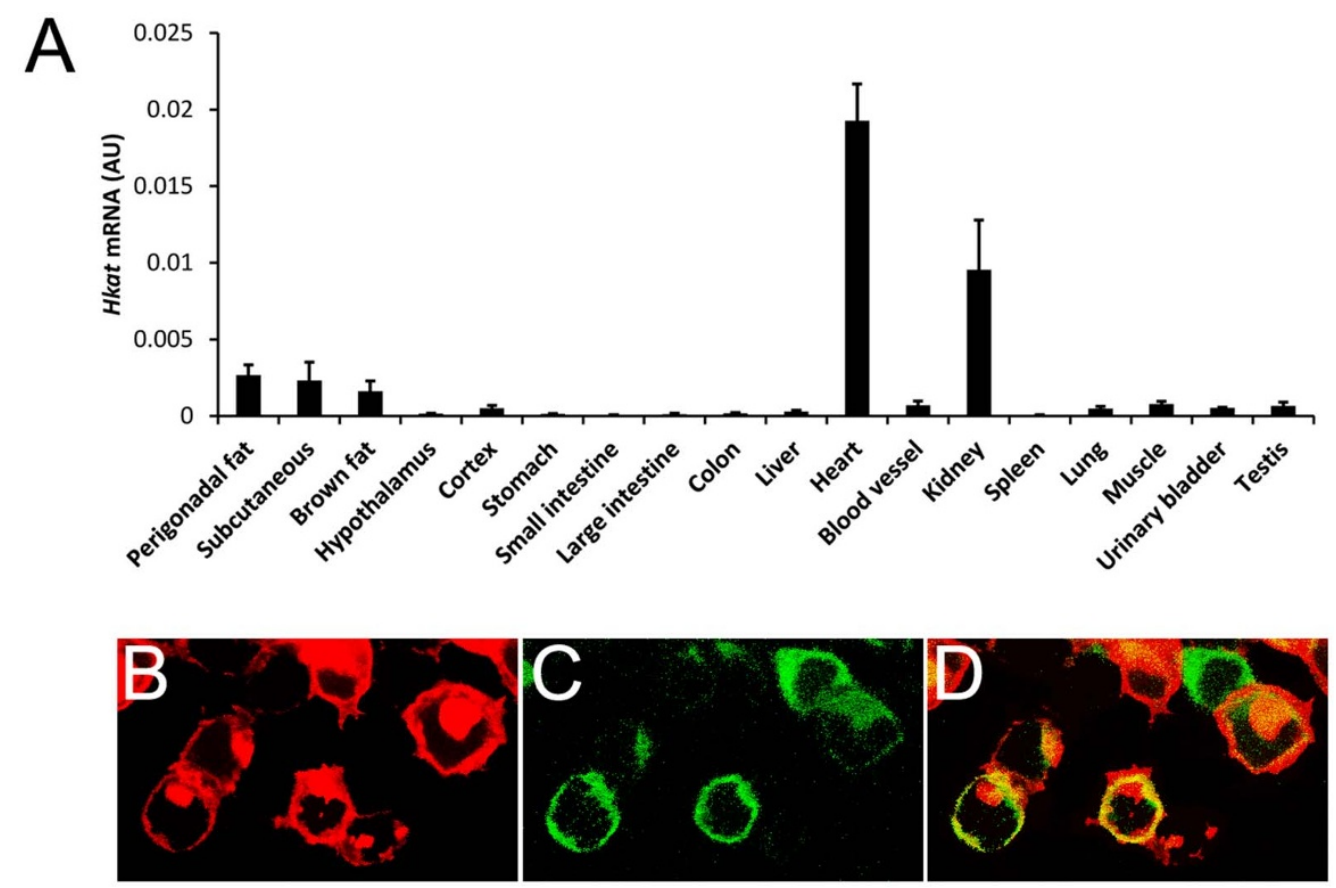

Figure 3 Hkat tissue expression pattern and subcellular localization. A) Hkat is expression in both white adipose tissue and brown adipose tissue, but is highest expressed in heart and kidney. B) Hkat is localized to the plasma membrane. Fluorescence imaging of HEK 293 cells transfected with plasmids encoding B) LCK-tRFP and C) Hkat-GFP and D) Merged picture.

$\operatorname{PPAR} \gamma$ agonists for 3 days to induce differentiation, followed by incubation in the regular maintenance medium for additional days. As a control, additional cells were also cultured in maintenance medium without differentiation. Ratio of Hkat expression between differentiated and non-differentiated cells was calculated (Fig. 4). Starting from day 2, Hkat had a more than 3 fold increase in differentiated cells, and reached about 7 fold on day 5 . Interestingly, on day 6 and 7, Hkat expression was reduced (Fig. 4). Therefore, Hkat was transiently induced during adipogenesis.

\section{Discussion}

We are now in the mist of a global obesity epidemic, because obesity is prevalent in both developed and developing countries, in both adults and children, and the increase in its prevalence continues ${ }^{34}$. Conclusive evidence suggests that obesity is associated with an increased risk of numerous health consequences, such as type 2 diabetes, coronary heart diseases, musculoskeletal diseases and mortality, and
Obesity is becoming one of the most significant contributors to ill health ${ }^{33,34}$. We are in urgent need to find novel therapeutic approaches to treat obesity and its complications. Proteins presented on the cell surface are accessible to various drug delivery mechanisms, therefore plasma membrane proteins are attractive drug targets $^{35}$. In that regard, Hkat is a potentially attractive drug target because it is localized to the plasma membrane.

The microarray technique has been routinely used to examine differential expression of genes from metabolic active organs, such as the liver, white adipose tissue (WAT) and brown adipose tissue (BAT), at different nutritional states, such as diet induced obesity and fasting ${ }^{36,37}$. In contrast, RNA-seq is newly developed sequencing based method to examine the expression of transcriptome. Compared to microarray, RNA-seq has many advantages. Two notable advantages are that RNA-seq is more sensitive, and identifies not only known genes, but also unknown transcripts ${ }^{38}$. We here used RNA-seq and identified Hkat as a novel nutritionally regulated genes in fat.



Figure $4 \mid$ Hkat is transiently induced during adipogenesis. 3T3 L1 preadipocytes were either differentiated into adipocytes or maintained in nondifferentiation medium, and Hkat expression was determined by qPCR in cells before (day 0) and after (day 1 to 7 ) starting differentiation. Shown are ratios of Hkat expression between cells with or without differentiation. Values for undifferentiated cells were normalized to 1 . $^{*}, \mathrm{P}<0.05 ; * *, \mathrm{P}<0.01$. 
There are some caveats in interpreting the results. First, although the current study focuses on the functions of Hkat in fat, Hkat is highest expressed in the heart and kidney. Therefore, it is likely that Hkat has important functions in the heart and kidney and these functions are not investigated in the current study. Second, the plasma membrane localization is shown by using an Hkat-GFP fusion protein expressed in HEK 293 cells, but it does not necessarily reflect its subcellular location in vivo, i.e., in cells of the heart, kidney and fat. To answer this question, using antibody against Hkat to do immunostaining on tissue sections will be much more informative. However, the antibody is not commercially available. Therefore, it is possible that in vivo, Hkat is localized to the membrane of organelles, not necessarily the plasma membrane. Third, Hkat harbors the conserved domain TraB, whose functions, however, could not be easily linked to adipocyte functions.

In summary, we have identified Hkat (Gm12824) as a novel nutritionally regulated gene in fat using RNA-seq. In WAT, both fasting and obesity dramatically reduce Hkat expression. In BAT, fasting reduces while obesity increases its expression. Being evolutionarily conserved, Hkat is localized to the plasma membrane and transiently induced during adipogenesis. The functional roles of Hkat and its conserved domain TraB in adipocytes, however, remain elusive.

\section{Methods}

Mice. Mice were housed at $22-24^{\circ} \mathrm{C}$ with a 14 -h light, 10 -h dark cycle and provided with ad libitum water and a chow diet (6\% calories from fat, 8664; Harlan Teklad, Indianapolis, IN) unless otherwise indicated. To examine nutritional stimulation induced gene expression, 10 4-week-old male C57B6 mice (Jackson laboratory, Bar Habor, ME) were placed on either a chow diet or a high-fat, high-sucrose diet $58 \%$ kcal from fat, 26\% kcal from sucrose, D-12331; Research Diets, New Brunswick, NJ) for 3 months. Five 8 -week-old mice were treated with 24 -hour fasting with 4 fed mice as controls. To examine the expression pattern of Hkat in various mouse tissues, 38 wk-old mice were used. All animal protocols were approved by the Animal Care and Use Committee of Wayne State University.

RNA extraction and quantitative real-time PCR. Dissected tissues were immediately placed into RNAlater solution (Ambion, Austin, TX) for subsequent RNA extraction. Total RNA was isolated from tissues with RNeasy tissue minikit with deoxyribonuclease treatment (QIAGEN, Valencia, CA). One microgram of RNA was reverse transcribed to cDNA using random hexamers (Superscript; Ambion). Relative expression levels were calculated and $\beta$-actin was used as an internal control. Primer sequences for Hkat were: forward, 5' -CCAGCCTACTTGTTTGGCAC-3'; reverse, $5^{\prime}$-GACTCGGGTATAGGGAACGTG- ${ }^{\prime}$. Primer sequences for $\beta$-actin were: forward, 5' -GTGACGTTGACATCCGTAAAGA-3'; reverse, 5' GCCGGACTCATCGTACTCC-3'.

Multiple alignments. Hkat protein IDs for Homo sapiens, Pan troglodytes, Macacamulatta, Canis lupus familiaris, Bos Taurus, Musmusculus, Rattusnorvegicus, Gallus gallus, Daniorerio, Caenorhabditiselegans were NP_001181915.1, XP_001142926.2, XP 001101255.2, XP_852692.2, XP_002686455.1, NP_001079018.1, XP_001072500.2, XP_422458.3, XP_694385.5, NP_510451.3, respectively. Hkat orthologs were based on HomoloGene Release 66, with HomoloGene ID 85034. The software MUSCLE ${ }^{39}$ was used to perform multiple alignments, and Blast ${ }^{40}$ was used to perform pairwise comparison between two sequences. ClustalW $2^{41}$ was used to generate phylogeny, and TreeDyn ${ }^{42}$ was used to visualize the tree.

Cell culture and adipocyte differentiation. 3T3-L1 cells (American Type Cell Collection, Manassask, VA) were maintained in Dulbecco's Modified Eagle Media (DMEM) containing $10 \%$ fetal bovine serum (FBS) at $37^{\circ} \mathrm{C}$ with $5 \% \mathrm{CO} 2$ in a humidified incubator. To differentiate 3T3-L1 cells into adipocytes, we used preadipocyte medium (Catalog number PM-1-L1, Zenbio, Research Triangle Park, NC), differentiation medium (DM-2-L1) and adipocyte maintenance medium (AM1-L1). Briefly, 3T3 L1 cells was allowed to reach confluence in preadipocytes medium (day -2), and after 48 hours (day 0 ), cells medium was changed to differentiation medium for 3 days (day 3 ), followed by incubating cells in adipocyte maintenance medium for additional days, with feeding cells every 2 days with the same medium.

Imaging. To examine Hkat subcellular localization, fusion protein of Hkat-GFP was made by cloning the Hkat open reading frame (Origene, MD) into the pCMV6-ACGFP vector. In HEK293 cells, the Hkat-GFP vector was cotransfected with a pCMV6 vector that encodes a fusion protein of LCK (lymphocyte-specific protein tyrosine kinase) and red fluorescence protein (Origene, MD) using Lipofectamine 2000 (Invitrogen, Grand Island, NY). Fluorescence Images were taken at the imaging core facility of Wayne State University with a Leica TCS SP5 Confocal Microscope.
Statistical analysis. Data are expressed as the mean \pm sem. Statistical significance was tested with unpaired two-tailed Student's $t$ tests. The differences were considered statistically significant if $\mathrm{P}<0.05$.

1. Attie, A. D. \& Scherer, P. E. Adipocyte metabolism and obesity. J Lipid Res $\mathbf{5 0}$ Suppl, S395-9 (2009).

2. Rosen, E. D. \& Spiegelman, B. M. Adipocytes as regulators of energy balance and glucose homeostasis. Nature 444, 847-53 (2006).

3. Kershaw, E. E. \& Flier, J. S. Adipose tissue as an endocrine organ. Journal of Clinical Endocrinology \& Metabolism 89, 2548-2556 (2004).

4. Spiegelman, B. M. \& Flier, J. S. Obesity and the regulation of energy balance. Cell 104, 531-43 (2001)

5. Flier, J. S. Obesity wars: molecular progress confronts an expanding epidemic. Cell 116, 337-50 (2004).

6. Barsh, G. S., Farooqi, I. S. \& O'Rahilly, S. Genetics of body-weight regulation. Nature 404, 644-51 (2000).

7. Zhang, Y. et al. Positional cloning of the mouse obese gene and its human homologue. Nature 372, 425-32 (1994).

8. Hu, E., Liang, P. \& Spiegelman, B. M. AdipoQ is a novel adipose-specific gene dysregulated in obesity. J Biol Chem 271, 10697-703 (1996).

9. Scherer, P. E., Williams, S., Fogliano, M., Baldini, G. \& Lodish, H. F. A novel serum protein similar to C1q, produced exclusively in adipocytes. J Biol Chem $\mathbf{2 7 0}$, 26746-9 (1995).

10. Maeda, K. et al. cDNA cloning and expression of a novel adipose specific collagenlike factor, apM1 (AdiPose Most abundant Gene transcript 1). Biochem Biophys Res Commun 221, 286-9 (1996).

11. Steppan, C. M. et al. The hormone resistin links obesity to diabetes. Nature 409, 307-12 (2001)

12. Hotamisligil, G. S. Inflammatory pathways and insulin action. Int J Obes Relat Metab Disord 27 Suppl 3, S53-5 (2003).

13. Kahn, B. B. \& Flier, J. S. Obesity and insulin resistance. Journal of Clinical Investigation 106, 473-81 (2000).

14. Taubes, G. As obesity rates rise, experts struggle to explain why. Science $\mathbf{2 8 0}$, 1367-8 (1998).

15. Allison, D. B., Fontaine, K. R., Manson, J. E., Stevens, J. \& VanItallie, T. B. Annual deaths attributable to obesity in the United States. JAMA 282, 1530-8 (1999).

16. Moitra, J. et al. Life without white fat: a transgenic mouse. Genes Dev 12,3168-81 (1998).

17. Shimomura, I. et al. Insulin resistance and diabetes mellitus in transgenic mice expressing nuclear SREBP-1c in adipose tissue: model for congenital generalized lipodystrophy. Genes Dev 12, 3182-94 (1998).

18. Ahima, R. S. \& Flier, J. S. Adipose tissue as an endocrine organ. Trends in Endocrinology and Metabolism 11, 327-332 (2000).

19. Mohamed-Ali, V., Pinkney, J. H. \& Coppack, S. W. Adipose tissue as an endocrine and paracrine organ. International Journal of Obesity 22, 1145-1158 (1998).

20. Trayhurn, P. \& Beattie, J. H. Physiological role of adipose tissue: white adipose tissue as an endocrine and secretory organ. Proceedings of the Nutrition Society $\mathbf{6 0}$, 329-339 (2001)

21. Fruhbeck, G., Gomez-Ambrosi, J., Muruzabal, F. J. \& Burrell, M. A. The adipocyte: a model for integration of endocrine and metabolic signaling in energy metabolism regulation. American Journal of Physiology-Endocrinology and Metabolism 280, E827-E847 (2001).

22. Flier, J. S. The Adipocyte - Storage Depot or Node on the Energy Information Superhighway. Cell 80, 15-18 (1995).

23. Lefterova, M. I. \& Lazar, M. A. New developments in adipogenesis. Trends Endocrinol Metab 20, 107-14 (2009).

24. Rosen, E. D. \& MacDougald, O. A. Adipocyte differentiation from the inside out. Nat Rev Mol Cell Biol 7, 885-96 (2006).

25. Rosen, E. D. \& Spiegelman, B. M. Molecular regulation of adipogenesis. Annu Rev Cell Dev Biol 16, 145-71 (2000).

26. Rosen, E. D., Walkey, C. J., Puigserver, P. \& Spiegelman, B. M. Transcriptional regulation of adipogenesis. Genes Dev 14, 1293-307 (2000).

27. MacDougald, O. A. \& Lane, M. D. Transcriptional regulation of gene expression during adipocyte differentiation. Annu Rev Biochem 64, 345-73 (1995).

28. Johnson, P. F. Molecular stop signs: regulation of cell-cycle arrest by C/EBP transcription factors. J Cell Sci 118, 2545-55 (2005).

29. Nerlov, C. The C/EBP family of transcription factors: a paradigm for interaction between gene expression and proliferation control. Trends Cell Biol 17, 318-24 (2007).

30. Rosen, E. D. et al. C/EBPalpha induces adipogenesis through PPARgamma: a unified pathway. Genes Dev 16, 22-6 (2002).

31. Tontonoz, P., Hu, E. \& Spiegelman, B. M. Stimulation of adipogenesis in fibroblasts by PPAR gamma 2, a lipid-activated transcription factor. Cell 79, 1147-56 (1994).

32. Popkin, B. M. \& Doak, C. M. The obesity epidemic is a worldwide phenomenon. Nutr Rev 56, 106-14 (1998).

33. Billington, C. J. et al. Overweight, obesity, and health risk. National Task Force on the Prevention and Treatment of Obesity. Arch Intern Med 160, 898-904 (2000).

34. WHO. Obesity: preventing and managing the global epidemic. Report of a WHO consultation. World Health Organ Tech Rep Ser 894, i-xii, 1-253 (2000).

35. Clark, H. F. et al. The secreted protein discovery initiative (SPDI), a large-scale effort to identify novel human secreted and transmembrane proteins: A bioinformatics assessment (vol 13, pg 2265, 2003). Genome Research 13, 2759-2759 (2003). 
36. Kennedy, A. R. et al. A high-fat, ketogenic diet induces a unique metabolic state in mice. Am J Physiol Endocrinol Metab 292, E1724-39 (2007).

37. Xu, H. Y. et al. Chronic inflammation in fat plays a crucial role in the development of obesity-related insulin resistance. Journal of Clinical Investigation 112, 1821-1830 (2003).

38. Gupta, R. K., Rosen, E. D. \& Spiegelman, B. M. Identifying Novel Transcriptional Components Controlling Energy Metabolism. Cell Metabolism 14, 739-745 (2011)

39. Edgar, R. C. MUSCLE: multiple sequence alignment with high accuracy and high throughput. Nucleic Acids Res 32, 1792-7 (2004).

40. Altschul, S. F. et al. Gapped BLAST and PSI-BLAST: a new generation of protein database search programs. Nucleic Acids Res 25, 3389-402 (1997).

41. Larkin, M. A. et al. Clustal W and Clustal X version 2.0. Bioinformatics 23, 2947-8 (2007).

42. Chevenet, F., Brun, C., Banuls, A. L., Jacq, B. \& Christen, R. TreeDyn: towards dynamic graphics and annotations for analyses of trees. BMC Bioinformatics 7 , 439 (2006)

\section{Acknowledgements}

The author thanks Fayi Yao and Zhiyao Fu for technical support and Dr. Abdul Abou-Samra for stimulating discussions. The present work was supported in part by a fund (176412) from Wayne State University to R.Z.

\section{Author contribution}

RZ designed the study, performed most of the experiments, analyzed data and wrote the manuscript. All authors reviewed the manuscript.

\section{Additional information}

Competing financial interests: The authors declare no competing financial interests.

License: This work is licensed under a Creative Commons

Attribution-NonCommercial-ShareAlike 3.0 Unported License. To view a copy of this license, visit http://creativecommons.org/licenses/by-nc-sa/3.0/

How to cite this article: Zhang, R. Hkat, a novel nutritionally regulated transmembrane protein in adipose tissues. Sci. Rep. 2, 825; DOI:10.1038/srep00825 (2012). 